\title{
CTLA4 methylation predicts response to anti-PD-1 and anti-CTLA-4 immunotherapy in melanoma patients
}

Diane Goltz, ${ }^{1}$ Heidrun Gevensleben, ${ }^{1}$ Timo J. Vogt, ${ }^{2}$ Joern Dietrich, ${ }^{2}$ Carsten Golletz, ${ }^{1}$ Friedrich Bootz, ${ }^{2}$ Glen Kristiansen, ${ }^{1}$ Jennifer Landsberg, ${ }^{3}$ and Dimo Dietrich ${ }^{2}$

Institute of Pathology, ${ }^{2}$ Department of Otolaryngology, Head and Neck Surgery, and ${ }^{3}$ Department of Dermatology and Allergy, University Hospital Bonn, Bonn, Germany.

Recent years have witnessed the groundbreaking success of immune checkpoint blockage (ICB) in metastasized malignant melanoma. However, biomarkers predicting the response to ICB are still urgently needed. In the present study, we investigated CTLA4 promoter methylation (mCTLA4) in $\mathbf{4 7 0}$ malignant melanoma patients from The Cancer Genome Atlas (non-ICB cohort) and in $\mathbf{5 0}$ individuals with metastasized malignant melanomas under PD-1/CTLA-4-targeted immunotherapy (ICB cohort). mCTLA4 levels were quantified using the Infinium HumanMethylation450 BeadChip (non-ICB cohort) and methylation-specific quantitative real-time PCR in DNA formalin-fixed and paraffin-embedded tissues (ICB cohort). Methylation levels were associated with molecular and clinicopathological variables and analyzed with respect to response (irRECIST) and overall survival. CTLA-4 mRNA and mCTLA4 showed a significant inverse correlation (non-ICB cohort: Spearman's $\rho=-0.416, P<0.001)$. In ICB-treated melanoma patients, low mCTLA4 was further strongly correlated with response to therapy $(P=\mathbf{0 . 0 0 9}$, ANOVA) and overall survival (hazard ratio $=2.06$ [95\% Cl: 1.29-3.29], $P=0.003$ ). Our data strongly support the assumption that $\mathrm{mCTLA4}$ predicts response to both anti-PD-1 and anti-CTLA- 4 targeted ICB in melanoma and provides paramount information for the selection of patients likely to respond to ICB.

Authorship note: DG and HG contributed equally to this work. JL and DD are co-senior authors.

Conflict of interest: DD owns patents (patents: DE 102016005 947.8, DE 10 2015009 187.5; patent applications: DE 102017 125 780.2, PCT/EP2016/001237, PCT/EP2017/061612) on methylation of immune checkpoint genes (including CTLA4) as predictive and prognostic biomarkers. DD is a consultant for $\mathrm{A}$ Innuscreen GmbH (Berlin, Germany), a $100 \%$ daughter company of Analytik Jena AG (Jena, Germany), and receives royalties from product sales (innuCONVERT kits)

Submitted: August 9, 2017

Accepted: May 23, 2018

Published: July 12, 2018

\section{Reference information:}

JCI Insight. 2018;3(13):e96793.

https://doi.org/10.1172/jci.

insight.96793.

\section{Introduction}

Immune checkpoint blockage (ICB) targeting the cytotoxic T lymphocyte-associated protein 4 (CTLA-4) or the programmed cell death 1 (PD-1) pathway, either alone or in combination, has proven efficacy in the treatment of various malignancies. Patients with metastatic malignant melanoma show high response rates with long-lasting tumor control in a subgroup of patients (1-4). ICB as adjuvant treatment in high-risk melanoma has further led to prolonged survival (5-7). Despite its remarkable success, resistance to primary therapy limits the therapeutic efficacy of ICB treatment. Moreover, ICB causes immune-related side effects requiring long-term medical management. Robust predictive biomarkers predicting responsiveness to ICB are therefore of paramount importance.

Research on predictive biomarkers for response to immunotherapies currently focuses on the expression of immune checkpoints, particularly PD-L1, mutational load and genomic stability, and immune cell infiltrates. Microsatellite instability and mismatch repair deficiency, for instance, have received FDA approval as predictive biomarkers for anti-PD-1 immunotherapy with pembrolizumab in microsatellite instability-high (MSI-H) or mismatch repair-deficient (dMMR) pediatric and adult solid tumors. The current understanding of response to ICB suggests that patients with a preexisting antitumor T cell response benefit from ICB. PD-L1 expression on tumor cells is associated with tumor-infiltrating T cells and can be induced by interferon (IFN) response signatures $(8,9)$. Therefore, numerous studies have evaluated PD-L1 expression on melanoma cells by immunohistochemistry, supporting a role as predictive biomarker. However, PD-L1-negative melanomas have also been shown to achieve durable clinical benefit from ICB, indicating the limited usefulness of PD-L1 expression as a robust biomarker (reviewed in ref. 10). A meta-analysis by Khunger et al. (11) of 6,664 patients with various malignancies including melanomas revealed an overall poor clinical performance of PD-L1 expression as a predictive biomarker for PD-1-targeted immunotherapies. 


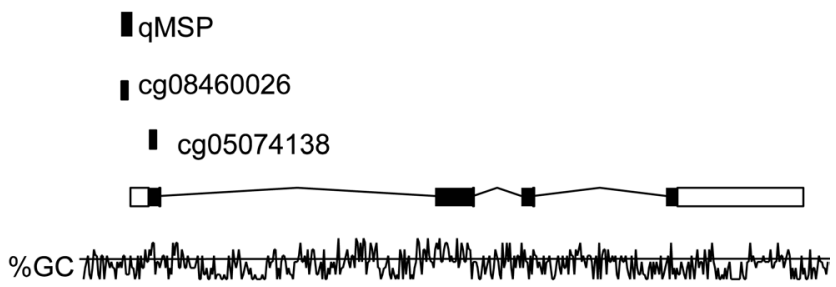

Figure 1. Genomic organization of the CTLA4 gene. Shown are GC density, introns, exons, and target sites of HumanMethylation450 BeadChip beads (cg08460026 and cg05074138) and qMSP assay. The modified illustration was exported from www.ensemble.org (version 89.38) and is based on Genome Reference Consortium Human Build 38 patch release 10 (GRCh38.p10).

In contrast to PD-1/PD-L1 expression, CTLA-4 expression on tumor cells has barely been investigated, and its role in melanomas remains unknown. In vitro, human melanoma cell lines show a high CTLA-4 expression regulated by the IFN- $\gamma / \mathrm{JAK} / \mathrm{STAT} 1-$ mediated signaling pathway (12). RNA sequencing (RNASeq) transcriptome data from 20 melanoma patients after anti-CTLA-4 immunotherapy revealed a correlation between an IFN- $\gamma$ response signature (including CTLA-4) and a long-term benefit from anti-CTLA-4 treatment. The authors consequently supported the notion that CTLA-4 might serve as a potential biomarker for the response to anti-CTLA-4 immunotherapy (12). In melanoma cells, CTLA-4 has also been identified as a direct transcriptional target of the Wnt/ $\beta$-catenin signaling pathway, and the activation of this pathway has been described as a tumor cell-intrinsic factor contributing to ICB resistance. Interestingly, $\beta$-catenin induces an increase in CTLA-4 mRNA and total CTLA-4 protein levels without concomitant changes in cell surface expression $(13,14)$. It has therefore been suggested that intracellular CTLA-4 might have a so far unknown function in melanoma cells. These data highlight the potential of CTLA-4 as a therapeutic target beyond its function in T cell biology. The fact that CTLA-4 expression in melanoma and immune cells is dynamically regulated in the tumor microenvironment hampers an accurate assessment. Additionally, tumor heterogeneity, sampling variability, and different assays and antibodies affect the accuracy of immune checkpoint expression analysis as potential biomarkers. In contrast to gene and protein expression, DNA methylation is a biologically and chemically more stable marker that can be accurately quantified, e.g., in formalin-fixed and paraffin-embedded (FFPE) tissues. Consequently, methylation serves as a biomarker for screening, diagnosis, prognosis, and prediction in various clinical settings (15-19). To better understand the regulation of CTLA-4 expression via promoter methylation in melanomas, we investigated $\mathrm{mCTLA4}$ and CTLA-4 expression in a large cohort of 470 melanoma patients with detailed clinicopathological follow-up and molecular characterization provided by The Cancer Genome Atlas (TCGA) (20). The utility of mCTLA4 as predictive biomarker for response to ICB was furthermore investigated retrospectively in a cohort of 50 metastatic melanoma patients treated with anti-PD-1 or/and anti-CTLA-4 antibodies.

\section{Results}

Association of CTLA4 methylation and mRNA expression (non-ICB cohort). Since aberrant promoter methylation is frequently observed in cancer leading to the activation or repression of genes, we analyzed the correlation between CTLA-4 mRNA expression and mCTLA4 in 468 malignant melanoma samples from the TCGA Research Network. Methylation assessed by 2 beads (cg08460026 and cg05074138) from the Infinium HumanMethylation450 BeadChip located within the transcriptional start site of CTLA4 (Figure 1) was included in the analysis. Mean methylation of the analyzed loci showed a significant inverse correlation with CTLA- 4 mRNA expression (Spearman's $\rho=-0.416, P<0.001$ ). Tumor content (percentage nuclei that are tumor cells) was $84.8 \%$ [95\% CI: 83.2-86.3]. Since mean methylation in the cohort was 43.1\% [95\% CI: 41.5-44.7] (Supplemental Table 1; supplemental material available online with this article; https://doi.org/10.1172/jci.insight.96793DS1), a subgroup of tumor cells are inevitably unmethylated. Since immune checkpoint genes are frequently coexpressed, we analyzed the correlation of CTLA-4 mRNA with PD-1 mRNA expression levels. Here, PD-1 and CTLA-4 mRNA were shown to be significantly coexpressed ( $\rho=0.579, P<0.001, n=468)$.

Association of CTLA4 methylation and $m R N A$ expression with immune cell infiltrates and IFN- $\gamma$ signature (non$I C B$ cohort). Clinicopathological parameters (e.g., tumor thickness, ulceration, absence of tumor infiltrating lymphocytes) and molecular features like $B R A F$ mutations are known prognostic factors in melanoma 
A

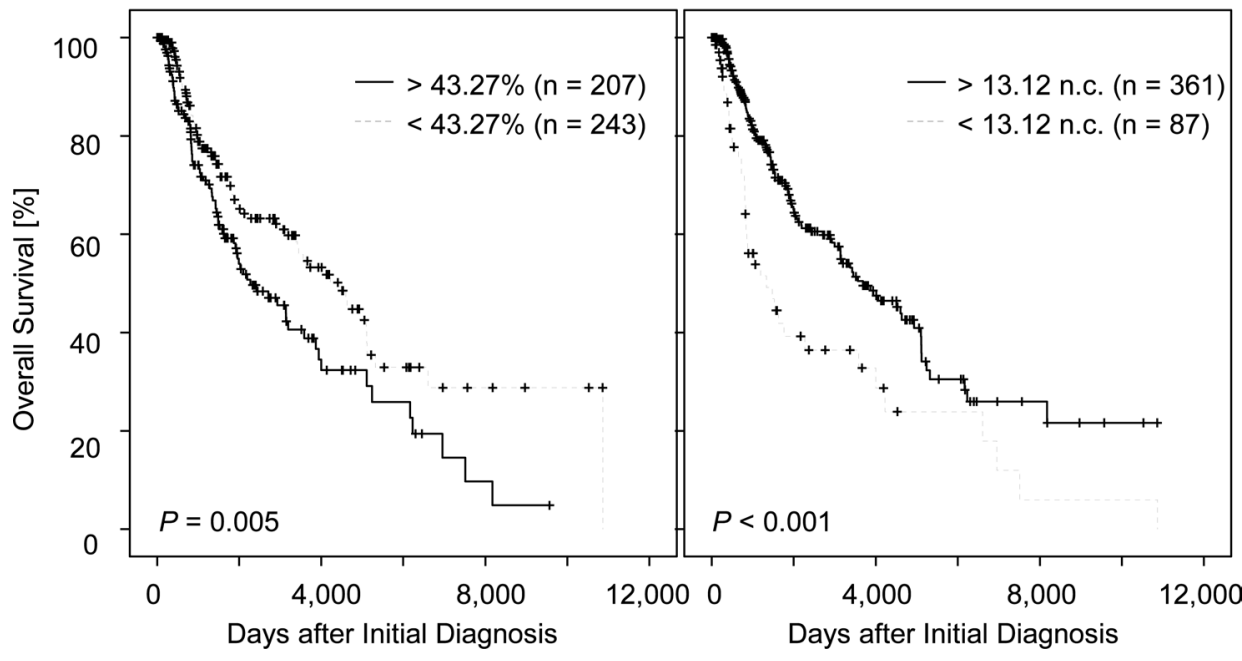

Figure 2. Kaplan-Meier analysis of overall survival in melanoma patients (non-ICB cohort) stratified according to CTLA4 methylation and mRNA expression. Patient samples were dichotomized based on optimized cutoffs (43.27\% methylation and 13.12 normalized mRNA counts [n.c.]). $P$ values refer to log-rank test.

patients. To identify established prognostic markers and additional parameters that correlate with CTLA-4 mRNA and mCTLA4, we performed a detailed analysis of clinicopathological parameters and their association with CTLA-4 mRNA expression and mCTLA4 (Supplemental Table 1). Of note, our analyses revealed significantly lower mCTLA4 levels in tumors with an activating BRAF mutation. As expected, increased CTLA-4 mRNA expression significantly correlated with lymphocyte scores $(\rho=0.328, P<0.001)$, and CTLA-4 mRNA expression was higher in samples with low tumor cell purity (correlation with sample purity score: $\rho=-0.479, P<0.001)$. In concordance, CTLA-4 mRNA expression significantly correlated with RNA signatures of immune cells (B cells, $\mathrm{CD} 4^{+}$and $\mathrm{CD} 8^{+} \mathrm{T}$ cells, neutrophils, macrophages, and dendritic cells) as provided by Li et al. (21) (Supplemental Table 1). Accordingly, a significant inverse correlation was found between $\mathrm{m} C T L A 4$ and $\mathrm{CD} 8^{+} \mathrm{T}$ cell and dendritic cell RNA signatures.

Since the infiltration of $\mathrm{CD} 8^{+} \mathrm{T}$ cells is associated with the activation of the IFN pathways and IFN- $\gamma$ / JAK/STAT1-mediated signaling pathways have been shown to induce CTLA-4 expression, we analyzed IFN- $\gamma /$ JAK/STAT1 pathway-associated genes with respect to CTLA-4 mRNA expression. CTLA-4 mRNA showed a significant positive correlation with the mRNA of IFN- $\gamma$ and the IFN- $\gamma$-regulated genes STAT1, STAT2, JAK2, and IRF9 (IFN- $\gamma$ : Spearman's $\rho=0.566$, STAT1: $\rho=0.462$, STAT2: $\rho=0.226$, JAK2: $\rho=0.336$, and IRF9: $\rho=0.352$; all $P<0.001, n=468)$. mCTLA4 correlated inversely with IFN- $\gamma$ mRNA expression $(\rho=-0.133, P=0.004, n=468)$.

Prognostic value of CTLA4 methylation and mRNA expression (non-ICB cohort). As CTLA-4 expression so far has not been tested as a prognostic factor, we investigated the prognostic impact of mCTLA4 and CTLA-4 mRNA expression in the non-ICB melanoma cohort. For survival analyses, death was considered the endpoint, and overall survival was defined as the interval from primary diagnosis to death. In Cox proportional hazards analysis, log2-transformed CTLA-4 mRNA showed significant association with overall survival (hazard ratio $[\mathrm{HR}]=0.92$ [95\% CI: 0.86-0.98], $P=0.009$ ). For log2-transformed $\mathrm{mCTLA4}$, no prognostic impact was shown (HR $=1.11$ [95\% CI: 0.86-1.44], $P=0.43$ ). For Kaplan-Meier $(\mathrm{KM})$ analysis, we further approximated optimized cutoffs for the categorization of patient samples as hyper- and hypomethylated. An optimized mCTLA4 cutoff (43.27\%) allowed for the stratification of patients into long-term and short-term overall survivors (likelihood ratio $[\mathrm{LH}]=7.76, P=0.005$ in KM [Figure 2A]; HR $=1.57$ [95\% CI: 1.14 2.16], $P=0.006$ in Cox proportional hazards analysis). Analogously, optimizing the CTLA-4 mRNA cutoff (13.12 normalized counts) confirmed the prognostic value of CTLA-4 mRNA expression ( $\mathrm{LH}=14.58, P<$ 0.001 in KM [Figure 2B]; HR $=0.50$ [95\% CI: 0.34-0.72], $P<0.001$ ). In concordance with the correlation of CTLA-4 and IFN- $\gamma$-associated gene expression and CD8 ${ }^{+} \mathrm{T}$ cell infiltrates, CTLA-4 mRNA expression was associated with a more favorable prognosis. However, the overall performance of mCTLA4 and CTLA-4 expression as a prognostic biomarker was rather weak, as indicated by relatively low HRs. 


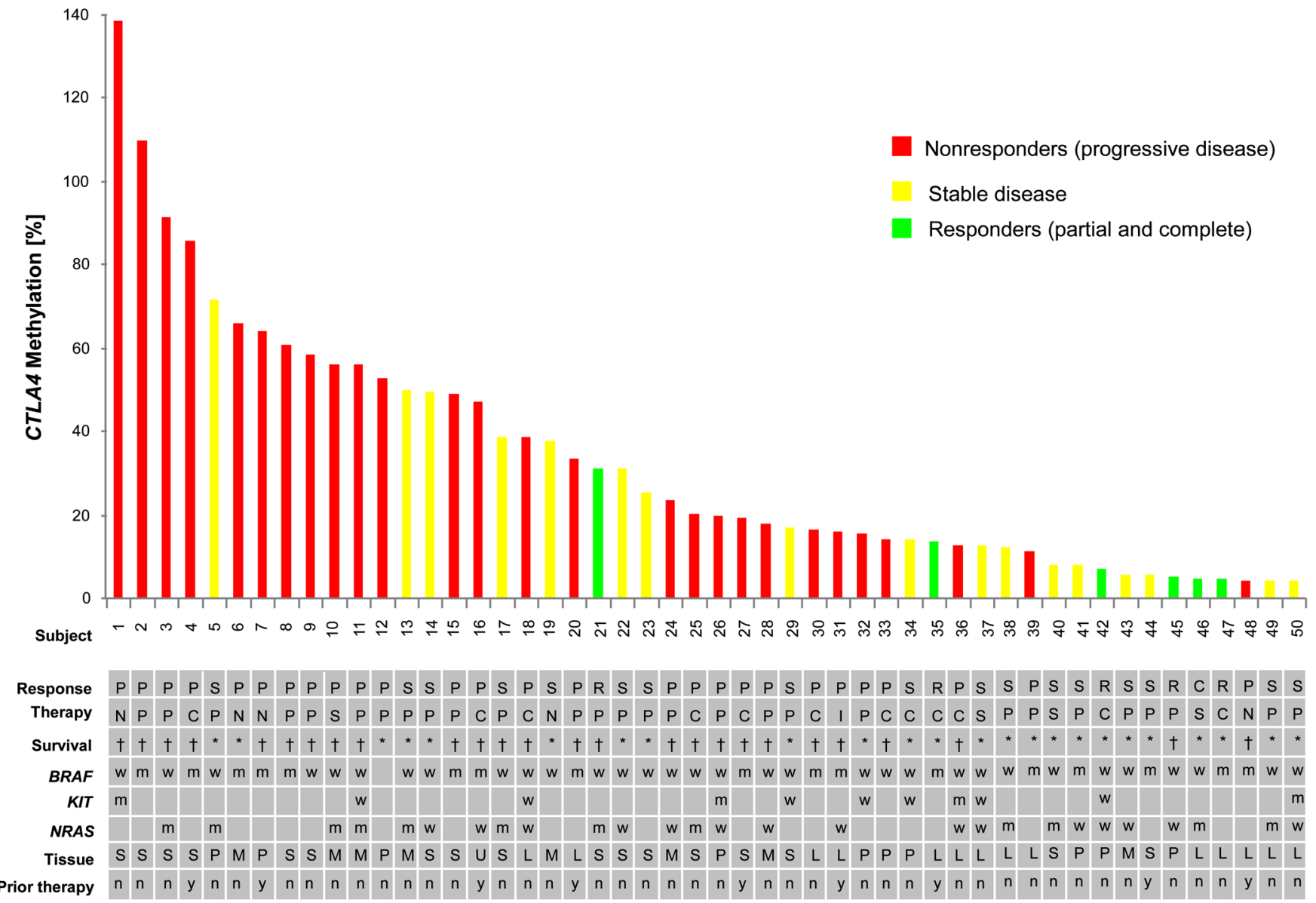

Figure 3. Clinical characteristics and CTL4A methylation of patients with metastatic melanoma in response to anti-PD-1/anti-CTLA-4 immunotherapy. Histogram showing qMSP quantification of the percentages of CTLA4 methylation versus characteristics of individual patients. $n=50$ patients. Response: P (progressive disease), S (stable disease), R (partial response), C (complete response), U (unknown); Therapy: P (pembrolizumab monotherapy), I (ipilimumab monotherapy), U (unknown), C (combined therapy [ipilimumab + nivolumab/pembrolizumab]), S (sequential therapy [ipilimumab + nivolumab/pembrolizumab]); Survival: †dead, *alive/censored; BRAF, KIT, NRAS mutations: w (wild type), m (mutated); Tissue: $\mathrm{S}$ (regional skin or soft tissue), P (primary disease), M (distant metastasis), U (unknown), L (regional lymph node); Prior therapy: $n$ (no prior BRAF/MEK therapy), y (prior BRAF/MEK therapy).

Association of CTLA4 methylation with response and survival in patients treated with anti-PD-1 and antiCTLA-4 immunotherapy (ICB cohort). The predictive value of $\mathrm{mCTLA4}$ for ICB was analyzed retrospectively in a cohort of 50 pretreatment melanoma samples. Mean mCTLA4 levels were slightly lower compared with the non-ICB cohort; however, variances may have resulted from different analytic techniques. CTLA4 mRNA was omitted from further analysis since the FFPE tissue quality did not allow for quantitative mRNA analysis. For detailed clinicopathological parameters and $\mathrm{mCTLA4}$ levels in the treatment cohort see Supplemental Table 2. We found significant differences in mean $\mathrm{mCTLA4}$ levels in tumors from patients with progressive disease (PD), stable disease (SD), and response to therapy (partial response [PR]/complete response [CR]; $P=0.009,1$-way ANOVA; Figures 3 and 4A). One-way ANOVA with Bonferroni's post hoc pairwise comparisons revealed a trend towards higher mCTLA4 in tumors from patients with $\mathrm{PD}$ compared with SD (mean difference $[\Delta]=21.2 \% \pm 8.5 \%, P=0.050$ ) and significant differences between $\mathrm{PD}$ and $\mathrm{PR} / \mathrm{CR}(\Delta=33.3 \% \pm 12.4 \%, P=0.031)$.

For survival analyses, death was considered the clinical endpoint, and overall survival was defined as the interval from primary dose of immune checkpoint inhibition to death. HR was first calculated using univariate Cox proportional hazards models based on log2-transformed $\mathrm{mCTLA4}$ levels without the introduction of a cutoff in order to avoid an overfitted model. Elevated $\mathrm{mCTLA4}$ levels were significantly associated with a higher risk of death $(\mathrm{HR}=2.06$ [95\% CI: 1.29-3.29], $P=0.003)$. We further performed $\mathrm{KM}$ with the log-rank test (LH). Patient samples were classified into $\mathrm{mCTLA4_{ \text {high } }}$ and $\mathrm{mCTLA4_{ \text {low } }}$ samples, 


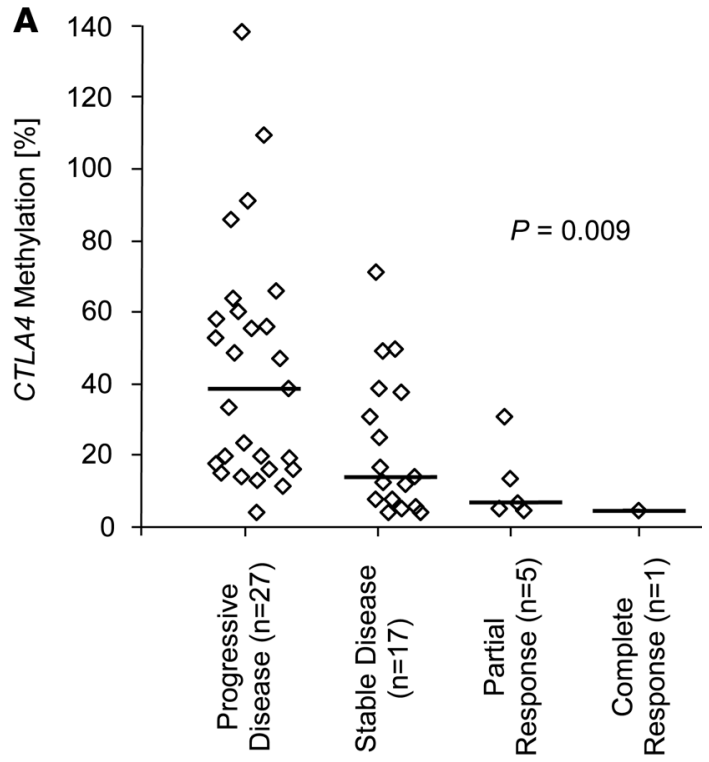

Response to Immunotherapy

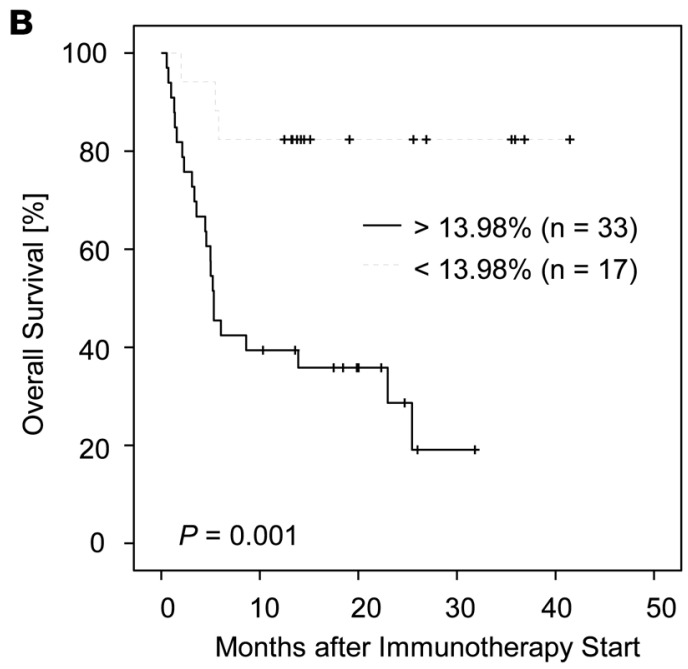

Figure 4. Association of CTLA4 methylation with response and survival in melanoma patients treated with immune checkpoint blockage. (A) CTLA4 DNA methylation in melanoma patient samples prior to immunotherapy. CTLA4 methylation (mCTLA4) was determined prior to immunotherapy, and patients were grouped according to irRECIST criteria. Low $\mathrm{mCTLA4}$ was associated with response to therapy. Bars indicate median methylation. $P$ value refers to ANOVA. (B) Overall survival under immunotherapy. Kaplan-Meier analysis of overall survival in 50 melanoma patients under immunotherapy stratified according to dichotomized mCTLA4. Patient samples were classified as mCTLA4-positive and -negative based on an optimized cutoff $(13.98 \%$ CTLA4 methylation). $P$ value refers to log-rank test.

respectively, based on an optimized cutoff (13.98\%). The prognostic value of categorized mCTLA4 showed significantly longer overall survival in patients with low promoter methylation $(\mathrm{LH}=11.23, P=0.001$ in $\mathrm{KM}$; Figure 4B). The Cox proportional hazards analysis revealed an $\mathrm{HR}=6.16$ [95\% CI: $1.84-20.7](P=$ 0.003 ) for patients with high mCTLA4 levels.

Significantly lower mCTLA4 levels were found in lymph node metastases compared with cutaneous or subcutaneous metastases ( $\Delta=35.7 \% \pm 9.8 \%, P=0.004$ by 1 -way ANOVA and Bonferroni's post hoc test; Supplemental Table 2). We performed survival subanalyses in these 2 sample groups in order to ensure that survival differences were associated with response to treatment and not type of metastasis. Analyzing $\mathrm{m} C T L A 4$ as continuous $\log 2$-transformed variable without dichotomization revealed a significantly worse survival in patients with higher methylation in both subcohorts with comparable HRs (lymph node metastases: $n=14, \mathrm{HR}=4.40$ [95\% CI: 1.06-18.33], $P=0.042$; cutaneous or subcutaneous metastases: $n=17$, $\mathrm{HR}=4.37[95 \%$ CI: $1.52-12.56], P=0.006)$.

\section{Discussion}

Biomarkers that predict response to ICB are urgently needed. In the present study, mCTLA4 was shown to be inversely correlated with CTLA-4 mRNA expression, suggesting an epigenetic regulation mechanism via 
DNA methylation in melanomas. Furthermore, increased mCTLA4 was a weak prognostic biomarker for poor overall survival in melanoma patients not treated with ICB, potentially indicating a higher ability of hypermethylated tumors for immune evasion. In patients under anti-PD-1 or combined anti-PD-1 and antiCTLA-4 ICB, however, mCTLA4 strongly predicted patients' survival and response to treatment. In accordance with our data, RNA-Seq transcriptome data of tumor tissue from 20 melanomas revealed that patients with a long-term survival benefit from anti-CTLA-4 treatment had significantly higher CTLA-4 expression levels compared with nonresponders (12). In this cohort, CTLA-4 expression was associated with the expression of IFN- $\gamma$ target genes (e.g., STAT1 and IRF1) and other immune checkpoints (e.g., PD-L1, TIM-3, and LAG-3). In the present study, we also identified an IFN- $\gamma$ response gene signature associated with high levels of $\mathrm{CD}^{+}$ T cell RNA signature and low mCTLA4 levels in the analyzed large non-ICB cohort. In both our study and the investigation conducted by Mo et al. (12), however, whole tumor tissue was used for analyses. It was therefore impossible to identify either melanoma cells or tumor-associated immune cells as the source of CTLA-4.

In the non-ICB cohort, on average $84.75 \%$ of nuclei were tumor nuclei while mean methylation was only $43.1 \%$. Accordingly, one or both CTLA4 alleles are unmethylated in a significant number of melanoma cells. Several studies have reported the expression of CTLA-4 in melanoma cell lines and melanoma tissue of patients $(12,13,22)$. Still, the effect of CTLA-4 expression in tumor cells remains barely understood. A potential functional role of CTLA-4 in melanoma cells might lie in the induction of caspasedependent apoptosis, which is induced following the interaction with CD80 or CD86 recombinant ligands in vitro (22). CTLA-4 overexpression in melanocytes was discovered in the context of erythemal inflammatory response in UVB-irradiated mouse skin, suggesting a survival mechanism upon DNA damage (23). CTLA-4 expression by tumor cells may also represent a mechanism by which tumors evolve to an immune evasion phenotype and inhibit adaptive and innate immunity. Tumor cell-intrinsic activation of $\beta$-catenin signaling can induce CTLA-4 expression and has been associated with a non-T cell-inflamed microenvironment and resistance to ICB (24). A direct biological link between mCTLA4 and immune evasion would support the assumption that $\mathrm{m} C T L A 4$ represents a truly predictive biomarker instead of a treatment-unrelated prognostic biomarker.

Apart from CTLA-4 expression in melanoma cells, CTLA-4 expression on immune cells appears to be functionally relevant for the response to ICB. Daud and colleagues recently used a novel multiparameter flow cytometry assay for the characterization of tumor-infiltrating immune cells in freshly isolated melanomas (25). The authors identified a unique immune cell population of partially exhausted cytotoxic CD8 ${ }^{+}$ $\mathrm{T}$ lymphocytes with a CTLA- $4^{\text {hi }} \mathrm{PD}-1^{\text {hi }}$ phenotype that robustly predicted the response to PD- 1 immune checkpoint inhibition. Pretherapeutic CTLA-4 expression on T cells was also recently found to be increased in responders to anti-PD-1 therapy compared with nonresponders using high-dimensional single-cell mass cytometry and a bioinformatics pipeline for the in-depth characterization of immune cell subsets in peripheral blood of melanoma patients (26).

$\mathrm{T}$ cell exhaustion is accompanied by stable epigenetic changes that limit the capability for reinvigoration through $\operatorname{ICB}(27,28)$. Epigenetic alterations, including DNA methylation changes, are a hallmark of $\mathrm{T}$ cell differentiation (29-31). De novo DNA methylation is involved in T cell exhaustion and limits ICBmediated $\mathrm{T}$ cell rejuvenation (32). Consequently, DNA methylation might also be suitable as a quantitative surrogate biomarker for T cell exhaustion. Since CTLA-4 expression has been shown to correlate inversely with promoter methylation in various malignancies (33-35), CTLA4 hypomethylation in tumors might be a surrogate biomarker for the state of $\mathrm{T}$ cell exhaustion. Such a second mechanism might add to the ability of $\mathrm{mCTLA} 4$ to assess the amount of CTLA-4-expressing and ICB-responding tumor cells, thereby explaining its high performance as a predictive biomarker.

Of interest, our data revealed a significant association of $\mathrm{mCTLA4}$ with $B R A F$ mutational status. This is consistent with the recently published observation that $B R A F^{V 600 E}$ - or $N R A S$-mutant cell lines expressed CTLA-4 at much greater levels than wild-type cell lines.

Combinatory therapies of ICB and BRAF inhibition have already proven efficacy in murine models (36) and anti-PD-1 or anti-CTLA-4 ICB is currently being tested together with BRAF inhibitors in several clinical trials. A potential crosstalk between CTLA-4 and MAPK signaling advocates testing $\mathrm{m} C T L A 4$ as a predictive biomarker for BRAF/MEK inhibitors and ICB combination therapies in melanomas with $B R A F$ mutation.

DNA methylation can be robustly and accurately determined even in limited amounts of FFPE tissues (37-39) and therefore constitutes a prime method for the assessment of biomarkers in human cancers. Furthermore, this methodology opens the possibility to assess methylation and mutation biomarkers at the 
same time. Most technologies applied to assess DNA methylation biomarkers depend on a previous deamination of DNA. Despite the reduced complexity of the DNA due to the conversion, DNA mutations can be detected. Even C-to-T transitions are preserved in either of the 2 DNA strands. Next-generation sequencing of bisulfite-converted DNA therefore might allow for the simultaneous testing of methylation and mutation biomarkers in the future. Additional genetic predictive biomarkers, i.e., tumor mutational burden, microsatellite instability (MSI), and mismatch repair (MMR) deficiency, could be multiplexed with methylation testing and tested in combination in various tumor entities. ICB is currently tested as combination therapy with PARP- and BRAF-/MEK-targeted therapies. Predictive biomarker tests for such combinatory therapies might include $M A P K$-pathway ( $R A S / R A F / M E K / E R K)$ and DNA repair enzyme (BRCAness) mutation analyses together with $C T L A 4$ methylation testing.

The present study is the first to our knowledge to demonstrate the predictive value of CTLA4 methylation in a treatment cohort of melanoma patients with long-term follow-up in response to immune checkpoint inhibition. Our study was conceptualized using a cohort of ICB-treated patients comprising anti-PD-1 monotherapy and sequential as well as combined anti-PD-1/anti-CTLA-4 ICB. Since the latter therapeutic regimes hold major effects on treatment responses, this inhomogeneity adds to the major weaknesses of this study. Methylation data, however, were obtained from pretreatment samples only, ensuring that treatment itself had no impact on $\mathrm{mCTLA4}$ value. Further studies analyzing matched pretherapeutic samples and samples obtained from tumors progressing under immunotherapy from the same patients are required to show that increasing $\mathrm{m} C T L A 4$ associates with adaptive therapy resistance.

Coexpression of immune checkpoint molecules frequently occurs on cancer-specific $\mathrm{T}$ cells (reviewed in ref. 40). In the present study, coexpression of PD-1 and CTLA-4 was shown in the non-ICB cohort and might provide a rationale for the predictive value of $\mathrm{mCTLA4}$ as a biomarker for single and combination therapies targeting PD-1 as well as CTLA-4. Consequently, we strongly recommend the prospective validation of $\mathrm{mCTLA} 4$ as a companion predictive biomarker for ICB in running clinical trials.

\section{Methods}

Patients (non-ICB cohort). A total of $n=470$ primary solid and metastatic malignant melanoma tissue samples provided by the TCGA (TCGA SKCM cohort) were included. Solid normal tissues and additional metastatic tumor tissues were excluded. Only 1 sample per patient was analyzed, and metastatic tumor tissue samples were favored in cases with more than 1 sample. Clinicopathological data were obtained from the TCGA Research Network. Molecular data were adopted from a previously published study (20). Clinicopathological data and molecular data are summarized in Supplemental Table 1. Sample purity and ploidy estimates provided by the TCGA Research Network were calculated using the ABSOLUTE algorithm (41). Quantitative data on infiltrating lymphocytes (B cells, dendritic cells, neutrophils, macrophages, $\mathrm{CD}^{+}$and $\mathrm{CD}^{+} \mathrm{T}$ cells) were adopted from Li et al. (21) who used an RNA-Seq signature as surrogate for immune cell infiltrates.

Data on lymphocyte distribution (0-3; $0=$ no lymphocytes within the tissue, $1=$ lymphocytes present involving $<25 \%$ of the tissue cross-sectional area, $2=1$ ymphocytes present in $25 \%$ to $50 \%$ of the tissue, $3=$ lymphocytes present in $>50 \%$ of tissue), lymphocyte density ( $0-3 ; 0=$ absent, $1=$ mild, $2=$ moderate, $3=$ severe), and lymphocyte ( $0-6$, score defined as the sum of the lymphocyte distribution and density scores) were adopted from the TCGA Research Network (20).

Patients (ICB cohort). A total of 50 patients diagnosed with metastasized melanomas and treated with checkpoint inhibition at the University Hospital Bonn between October 2014 and April 2017 were included in the cohort. Pretreatment samples of 10 primary tumors, 17 cutaneous or subcutaneous metastases, 14 lymph node metastases, and 8 distant metastases were analyzed. Of the 50 melanoma patients included in the study, 17 patients harbored $B R A F$ mutations, 32 individuals were $B R A F$ wild type, and the mutational status of 1 patient was unknown. The majority of patients received therapeutic regimens with anti-PD-1 inhibition (Supplemental Table 2). In case of adverse effects, the regimen was switched to anti-CTLA-4 inhibition. One patient received ipilimumab as monotherapy. Response patterns were reported based on immune-related response criteria (irRECIST). Altogether, 27 patients (54\%) suffered from PD, 17 patients presented with SD (34\%), 5 patients had PR (10\%), and 1 patient achieved CR under therapy $(2 \%)$.

$m R N A$ expression analysis (non-ICB cohort). mRNA data provided by the TCGA Research Network (http://cancergenome.nih.gov/) were generated by means of the Illumina HiSeq 2000 RNA Sequencing 
Version 2 analysis (Illumina, Inc.). Level 3 expression data were downloaded from the TCGA web page. Normalized counts per genes were calculated using the SeqWare framework via the RSEM algorithm (42). Data on mRNA expression were available from $n=468$ samples.

Methylation analysis (non-ICB cohort). Gene methylation data generated by the TCGA Research Network were downloaded from the UCSC Xena browser (www.xena.ucsc.edu). TCGA methylation analysis was performed using the Infinium HumanMethylation450 BeadChip (Illumina, Inc.). Methylation levels ( $\beta$ values $)$ were calculated as $\beta$ value $=($ Intensity_Methylated $) /($ Intensity_Methylated + Intensity_Unmethylated $+\alpha$ ) (43). The constant offset $\alpha$ was set to 0 . The $\beta$ values (values between 0 and 1 ) were multiplied with the factor $100 \%$ in order to show percentage methylation (0 to 100\%). Two beads (cg08460026 and cg05074138) targeting loci within the transcriptional start site of CTLA4 (Figure 1) were investigated. Mean $\beta$ values from both beads were computed for each patient sample. Methylation data were procurable from all $n=470$ included patient samples.

Methylation analysis (ICB cohort). The InnuCONVERT Bisulfite All-In-One Kit (Analytik Jena) was used to prepare bisulfite DNA from FFPE tissues. mCTLA4 was quantified by means of a methylation-specific real-time mCTLA4/ ACTB duplex PCR (qMSP) assay (mCTLA4 oligonucleotides: 6-FAMAAGTCGTGGGTTTAGTTGTTAC-BHQ-1, probe; GTTTTTTTGTTTTGGTTTTACGA, reverse primer; TACTTAAAATTATCTTTTCGACG, forward primer) under PCR buffer and cycling conditions as described previously (44).

Statistics. Statistical analyses were conducted using SPSS, version 23.0 (SPSS Inc.). Correlations were calculated using Spearman's rank correlation (Spearman's $\rho$ ). Mean value comparisons were performed with Wilcoxon-Mann-Whitney $U$ (2 groups) or Kruskal-Wallis (> 2 groups) test. Multiple comparisons between groups were further tested with 1-way ANOVA and Bonferroni's post hoc test. Survival analyses were performed using the KM method, likelihood ratios, and Cox proportional hazards regression. $P$ values refer to log-rank and Wald tests. For KM analysis, methylation levels and mRNA expression levels were dichotomized based on an optimized cutoff. Cox proportional hazards analyses were performed with dichotomized (based on optimized cutoffs) and continuous methylation and mRNA expression data (log2transformed). Two-sided $P$ values $<0.05$ were considered statistically significant.

Study approval. The study was approved by the Institutional Review Board at the University Hospital of Bonn (vote no. 187/16).

\section{Author contributions}

DG, HG, JL, and DD designed the study, analyzed data, and wrote the manuscript. TJV and JD analyzed and acquired data. CG conducted the experiments. FB, GK, and JL provided patient samples.

\section{Acknowledgments}

This work was supported by the Bonn NeuroImmunology (BonnNi) program funded by the Else KrönerFresenius Stiftung under grant Q-611.2354 given to T.J. Vogt and J. Landsberg.

Address correspondence to: Dimo Dietrich, University Hospital Bonn, Department of Otolaryngology, Head and Neck Surgery, Sigmund-Freud-Strasse 25, 53105 Bonn, Germany. Phone: 49.228.287.13702; Email: dimo.dietrich@gmail.com.

1. Wolchok JD, et al. Nivolumab plus ipilimumab in advanced melanoma. N Engl J Med. 2013;369(2):122-133

2. Le DT, et al. PD-1 blockade in tumors with mismatch-repair deficiency. N Engl J Med. 2015;372(26):2509-2520.

3. Hodi FS, et al. Combined nivolumab and ipilimumab versus ipilimumab alone in patients with advanced melanoma: 2-year overall survival outcomes in a multicentre, randomised, controlled, phase 2 trial. Lancet Oncol. 2016;17(11):1558-1568.

4. Robert C, et al. Pembrolizumab versus ipilimumab in advanced melanoma. N Engl J Med. 2015;372(26):2521-2532.

5. Weber J, et al. Adjuvant nivolumab versus ipilimumab in resected stage III or IV melanoma. N Engl J Med. 2017;377(19):1824-1835.

6. Eggermont AM, et al. Adjuvant ipilimumab versus placebo after complete resection of high-risk stage III melanoma (EORTC 18071): a randomised, double-blind, phase 3 trial. Lancet Oncol. 2015;16(5):522-530.

7. Eggermont AM, et al. Prolonged survival in stage III melanoma with ipilimumab adjuvant therapy. $N$ Engl J Med. 2016;375(19):1845-1855.

8. Tumeh PC, et al. PD-1 blockade induces responses by inhibiting adaptive immune resistance. Nature. 2014;515(7528):568-571

9. Bald T, et al. Immune cell-poor melanomas benefit from PD-1 blockade after targeted type I IFN activation. Cancer Discov. 2014;4(6):674-687.

10. Nishino M, Ramaiya NH, Hatabu H, Hodi FS. Monitoring immune-checkpoint blockade: response evaluation and biomarker 
development. Nat Rev Clin Oncol. 2017;14(11):655-668.

11. Khunger M, et al. Programmed cell death 1 (PD-1) ligand (PD-L1) expression in solid tumors as a predictive biomarker of benefit from PD-1/PD-L1 axis inhibitors: A systematic review and meta-analysis. JCO Precis Oncol. 2017;(1):1-15.

12. Mo X, et al. Interferon- $\gamma$ signaling in melanocytes and melanoma cells regulates expression of CTLA-4. Cancer Res. 2018;78(2):436-450.

13. Shah KV, Chien AJ, Yee C, Moon RT. CTLA-4 is a direct target of Wnt/beta-catenin signaling and is expressed in human melanoma tumors. J Invest Dermatol. 2008;128(12):2870-2879.

14. Sharma P, Hu-Lieskovan S, Wargo JA, Ribas A. Primary, adaptive, and acquired resistance to cancer immunotherapy. Cell. 2017;168(4):707-723.

15. Esteller M, et al. Inactivation of the DNA-repair gene MGMT and the clinical response of gliomas to alkylating agents. $N E n g l J$ Med. 2000;343(19):1350-1354

16. Church TR, et al. Prospective evaluation of methylated SEPT9 in plasma for detection of asymptomatic colorectal cancer. Gut. 2014;63(2):317-325

17. Partin AW, et al. Clinical validation of an epigenetic assay to predict negative histopathological results in repeat prostate biopsies. J Urol. 2014;192(4):1081-1087.

18. van Kessel KE, et al. Validation of a DNA methylation-mutation urine assay to select patients with hematuria for cystoscopy. J Urol. 2017;197(3 Pt 1):590-595.

19. Imperiale TF, et al. Multitarget stool DNA testing for colorectal-cancer screening. N Engl J Med. 2014;370(14):1287-1297.

20. Cancer Genome Atlas Network. Genomic classification of cutaneous melanoma. Cell. 2015;161(7):1681-1696.

21. Li B, et al. Comprehensive analyses of tumor immunity: implications for cancer immunotherapy. Genome Biol. 2016;17(1):174

22. Contardi E, et al. CTLA-4 is constitutively expressed on tumor cells and can trigger apoptosis upon ligand interaction. Int J Cancer. 2005;117(4):538-550.

23. Zaidi MR, et al. Interferon- $\gamma$ links ultraviolet radiation to melanomagenesis in mice. Nature. 2011;469(7331):548-553.

24. Spranger S, Bao R, Gajewski TF. Melanoma-intrinsic $\beta$-catenin signalling prevents anti-tumour immunity. Nature. 2015;523(7559):231-235.

25. Daud AI, et al. Tumor immune profiling predicts response to anti-PD-1 therapy in human melanoma. J Clin Invest. 2016;126(9):3447-3452.

26. Krieg C, et al. High-dimensional single-cell analysis predicts response to anti-PD-1 immunotherapy. Nat Med. 2018;24(2):144-153.

27. Pauken KE, et al. Epigenetic stability of exhausted T cells limits durability of reinvigoration by PD-1 blockade. Science. 2016;354(6316):1160-1165.

28. Sen DR, et al. The epigenetic landscape of T cell exhaustion. Science. 2016;354(6316):1165-1169.

29. Durek P, et al. Epigenomic profiling of human $\mathrm{CD}^{+} \mathrm{T}$ cells supports a linear differentiation model and highlights molecular regulators of memory development. Immunity. 2016;45(5):1148-1161.

30. Scharer CD, Barwick BG, Youngblood BA, Ahmed R, Boss JM. Global DNA methylation remodeling accompanies CD8 T cell effector function. J Immunol. 2013;191(6):3419-3429.

31. Youngblood B, et al. Effector CD8 T cells dedifferentiate into long-lived memory cells. Nature. 2017;552(7685):404-409.

32. Ghoneim HE, et al. De novo epigenetic programs inhibit PD-1 blockade-mediated T cell rejuvenation. Cell. 2017;170(1):142-157.e19.

33. Marwitz S, Scheufele S, Perner S, Reck M, Ammerpohl O, Goldmann T. Epigenetic modifications of the immune-checkpoint genes CTLA4 and PDCD1 in non-small cell lung cancer results in increased expression. Clin Epigenetics. 2017;9:51.

34. Goltz D, et al. PD-L1 (CD274) promoter methylation predicts survival in patients with acute myeloid leukemia. Leukemia 2017;31(3):738-743

35. Röver LK, et al. PD-1 (PDCD1) promoter methylation is a prognostic factor in patients with diffuse lower-grade gliomas harboring isocitrate dehydrogenase (IDH) mutations. EBioMedicine. 2018;28:97-104.

36. Cooper ZA, et al. Response to BRAF inhibition in melanoma is enhanced when combined with immune checkpoint blockade Cancer Immunol Res. 2014;2(7):643-654.

37. Uhl B, et al. PITX2 DNA methylation as biomarker for individualized risk assessment of prostate cancer in core biopsies. $J M o l$ Diagn. 2017;19(1):107-114.

38. Dietrich D, et al. Analysis of DNA methylation of multiple genes in microdissected cells from formalin-fixed and paraffinembedded tissues. J Histochem Cytochem. 2009;57(5):477-489.

39. Dietrich D, et al. Development and clinical validation of a real-time PCR assay for PITX2 DNA methylation to predict prostatespecific antigen recurrence in prostate cancer patients following radical prostatectomy. J Mol Diagn. 2013;15(2):270-279.

40. Nirschl CJ, Drake CG. Molecular pathways: coexpression of immune checkpoint molecules: signaling pathways and implications for cancer immunotherapy. Clin Cancer Res. 2013;19(18):4917-4924.

41. Carter SL, et al. Absolute quantification of somatic DNA alterations in human cancer. Nat Biotechnol. 2012;30(5):413-421.

42. Li B, Dewey CN. RSEM: accurate transcript quantification from RNA-Seq data with or without a reference genome. BMC Bioinformatics. 2011;12:323.

43. Du P, et al. Comparison of Beta-value and M-value methods for quantifying methylation levels by microarray analysis. $B M C$ Bioinformatics. 2010;11:587.

44. Gevensleben $\mathrm{H}$, et al. PD-L1 promoter methylation is a prognostic biomarker for biochemical recurrence-free survival in prostate cancer patients following radical prostatectomy. Oncotarget. 2016;7(48):79943-79955. 